\title{
A revisão da Política Nacional de Atenção Básica e a Promoção da Atividade Física
}

\section{Mathias Roberto Loch1,2; Emmanuelly Correia de Lemos,3,; Fernando Vinholes Siqueira,5; Luiz Augusto Facchini}

São inegáveis os avanços da atividade física no Brasil, especialmente a partir dos anos 2000, não apenas na pesquisa, mas também na vigilância e na sua participação no Sistema Único de Saúde (SUS) por meio de diversas políticas públicas ${ }^{1}$. Neste contexto é fundamental considerar a Política Nacional de Atenção Básica (PNAB), que é a base para diversas outras ações e programas de saúde.

A PNAB foi aprovada em $2006^{2}$, tendo como marco a consolidação e qualificação da Estratégia Saúde da Família (ESF), e revisada em 2011 para fortalecer os avanços obtidos e qualificar o papel de centro ordenador das redes de atenção à saúde. Esta primeira revisão foi importante, pois possibilitou a ampliação das ações intersetoriais e de promoção da saúde, inclusive a partir de Programas como o Academia da Saúde e o Saúde na Escola³.

Em 2017 foi aprovada uma nova revisão da $\mathrm{PNAB}^{4}$, criticada por importantes entidades ligadas ao movimento sanitário brasileiro, como por exemplo, a Associação Brasileira de Saúde Coletiva (ABRASCO), o Centro Brasileiro de Estudos da Saúde (CEBES) e a Escola Nacional de Saúde Pública Sergio Arouca (ENSP/

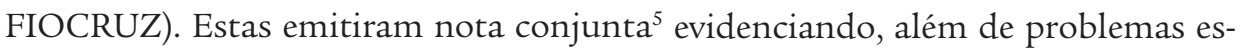
pecíficos de concepção da nova $\mathrm{PNAB}$, um cenário de grande instabilidade democrática, retirada de direitos sociais e de redução de investimentos públicos. Assim, explicitaram o temor de que a PNAB revisada ameace os princípios finalísticos do SUS (universalidade, equidade e integralidade).

Uma das principais mudanças na nova versão da PNAB diz respeito à possibilidade de financiamento a outros formatos de equipe, utilizando recursos do Piso da Atenção Básica Variável, que eram destinados originalmente para incentivar a implantação e manutenção da ESF. O argumento a favor de tal mudança é que estas irão permitir que os municípios se adequem às restrições orçamentárias, de acordo com as características e necessidades locais. No entanto, muitos acreditam que isso poderá acabar com a prioridade do modelo da ESF na organização da rede básica de saúde e do SUS, uma vez que abre a possibilidade de que outros modelos de atenção sejam implementados, podendo-se inclusive reduzir o escopo da Atenção Básica. Com esta nova realidade, entendemos que haverá um importante

1 Universidade Estadual de Londrina, Departamento de Educação Física, Paraná, Brasil. 2 Universidade Estadual de Londrina, Programa de Pós Graduação em Saúde Coletiva, Paraná, Brasil.

3 Universidade Estadual de Pernambuco/Universidade Federal da Paraíba, Programa Associado de Pós Graduação em Educação Física. 4 Secretaria Estadual de Saúde de Pernambuco, Pernambuco, Brasil.

5 Universidade Federal de Pelotas. Escola Superior de Educação Física. Programa de Pós Graduação em Educação Física, Rio Grande do Sul, Brasil.

6 Universidade Federal de Pelotas. Faculdade de Medicina, Departamento de Medicina Social, Mestrado Profissional em Saúde da Família, PROFSAÚDE/UFPel.

7 Universidade Federal de Pelotas. Faculdade de Medicina, Departamento de Medicina Social, Rio Grande do Sul, Brasil. 
retrocesso, pois acabarão por surgir modelos de atenção limitados aos aspectos biomédicos e clínicos, em detrimento à um entendimento mais amplo do processo saúde-doença, que consideramos muito mais adequado para o real enfrentamento da complexa situação epidemiológica brasileira.

Não descartamos que a mudança da nomenclatura do NASF (criados como "Núcleos de Apoio à Saúde da Família" e agora denominados "Núcleo Ampliado de Saúde da Família e Atenção Básica”) vá além de uma simples mudança de nomenclatura e sinalize a tendência à um novo direcionamento das ações do NASF, passando a privilegiar a atuação específica de cada profissão, ao invés de um olhar ampliado e interprofissional. Neste sentido, resgatar o sentido do princípio da Integralidade no SUS, nos parece ser fundamental e urgente.

Importante chamar a atenção para o fato de que a revisão na PNAB não aconteceu como ato "isolado". Ao contrário, ela parece muito bem articulada com uma série de outros acontecimentos e ações políticas, que, grosso modo, buscam diminuir a participação do Estado e fortalecer o setor privado. Talvez a ameaça mais explícita de sucateamento do SUS seja a Emenda Constitucional 956, que congelou os investimentos públicos por 20 anos. Tal Emenda é de grande gravidade e determinará sérias implicações na organização dos serviços de saúde brasileiros e, consequentemente, nos indicadores de saúde da população. Faz se necessário lembrar que o SUS tem sido, desde sua implementação, subfinanciado e que com o envelhecimento da população brasileira os recursos para a saúde, que já são insuficientes, serão ainda mais escassos. Esperamos que tal absurdo, o congelamento nos investimentos em atividades que são "fins" do Estado por um período tão longo, sejam revistos o mais rapidamente possível.

O cenário é complexo e certamente as iniciativas relacionadas a promoção da atividade física podem ser muito afetadas. Apesar de estarem bem estabelecidas as relações entre a prática de atividade física e importantes indicadores de saúde, os parcos recursos da área da saúde poderão negligenciá-la. Em consequência do baixo investimento em promoção da atividade física poderá haver um aumento a médio e longo prazo nos gastos com tratamento de doenças crônicas, por exemplo. Evidentemente que não se trata aqui de considerar que a atividade física é o único fator relacionado as doenças e condições crônicas, nem reforçar o olhar limitado que considera a atividade física importante para a vida humana somente pelo seu potencial papel na prevenção e tratamento de diversas doenças crônicas. Nunca é demais ressaltar que a prevenção de doenças (e em um sentido ainda mais amplo, a promoção da saúde) é complexa e deve englobar diversas ações, não se limitando ao setor saúde. Assim, ações amplas e articuladas, intra e intersetorias se fazem necessárias e podem ser tão diversas quanto o aconselhamento para a prática de atividade física até políticas que gerem desenvolvimento econômico e social e diminuam as nossas diversas desigualdades, além da garantia de acesso universal aos serviços de saúde.

Dado esta realidade, ressaltamos que a promoção da atividade física tem potencialmente um papel relevante no enfrentamento do quadro epidemiológico brasileiro. Ademais, consideramos que para que a promoção da atividade física seja efetiva, são necessárias ações que estejam plenamente alinhadas com a PNAB e muito nos preocupa as modificações que foram realizadas, pelos motivos 
já apontamos anteriormente. Assim, enfatizamos que todos os atores que estão de algum modo envolvidos com a complexa tarefa de promover atividade física, desde os profissionais que atuam na Atenção Básica até pesquisadores e gestores desta área, precisam compreender que mudanças em políticas estratégicas como a PNAB tem potencialmente um impacto importante na sua especificidade. Entendemos que a área deve buscar uma mobilização e participação mais ativa e efetiva, evitando inclusive perder o espaço conquistado nos últimos anos, que inclusive foi traduzido em benefícios para população. Certamente há muito que se evoluir na promoção da atividade física no Brasil, e isso passa, no nosso entendimento, necessariamente pelo da Atenção Básica e pela defesa incondicional dos princípios finalísticos do SUS.

\section{Conflito de interesses}

Os autores declaram não haver conflito de interesses.

\section{Contribuiç̧ão dos autores}

Loch MR, concebeu a ideia do texto e escreveu a versão inicial do manuscrito. Lemos EC, Siqueira FV e Facchini, LA, realizaram revisão crítica do manuscrito e contribuíram com novos argumentos que foram incorporados na versão final do texto.

\section{Referências}

1. Hallal PC. Atividade física e saúde no Brasil: pesquisa, vigilância e políticas. Cad. Saúde Pública. 2014;30(12):2487-89.

2. Brasil. Ministério da Saúde. Secretaria de Atenção à Saúde. Departamento de Atenção Básica. Política nacional de atenção básica / Ministério da Saúde, Secretaria de Atenção à Saúde, Departamento de Atenção à Saúde. - Brasília: Ministério da Saúde, 2006. Disponível em: http://bvsms.saude.gov.br/bvs/publicacoes/politica_nacional_atencao_ basica_2006.pdf. Acesso em 25 de fev. 2018.

3. Brasil. Ministério da Saúde. Secretaria de Atenção à Saúde. Departamento de Atenção Básica. Política Nacional de Atenção Básica / Ministério da Saúde. Secretaria de Atenção à Saúde. Departamento de Atenção Básica. - Brasília: Ministério da Saúde, 2012. Disponível em: < http://189.28.128.100/dab/docs/publicacoes/geral/pnab.pdf>. Acesso em 25 de fev. 2018.

4. Brasil. Portaria no 2.436 de 21 de setembro de 2017. Aprova a Política Nacional de Atenção Básica, estabelecendo a revisão de diretrizes para a organização da Atenção Básica, no âmbito do Sistema Único de Saúde (SUS). Diário Oficial da União 2017. Disponível em: http://pesquisa.in.gov.br/imprensa/jsp/visualiza/index. jsp?jornal=1\&pagina=68\&data=22/09/2017. Acesso em 25 de fev. 2018.

5. Associação Brasileira de Saúde Coletiva; Centro Brasileiro de Estudos de Saúde; Escola Nacional de Saúde Pública Sérgio Arouca. Contra a reformulação da PNAB - nota sobre a revisão da Política Nacional de Atenção Básica. Disponível em: https://www.abrasco.org. $\mathrm{br} /$ site/noticias/posicionamentos-oficiais/contra-reformulacao-da-pnab-nota-sobrerevisao-da-politica-nacional-de-atencao-basica/29798/). Acesso em 25 fev. 2018. 
6. Brasil. Constituição (1988). Emenda Constitucional no 95, de 15 de dezembro de 2016. Altera o Ato das Disposições Constitucionais Transitórias, para instituir o Novo Regime Fiscal, e dá outras providências. Disponível em: http://www2.camara. leg.br/legin/fed/emecon/2016/emendaconstitucional-95-15-dezembro-2016-784029publicacaooriginal-151558-pl.html. Acesso em 25 de fev. 2018.

ENDEREÇO PARA

CORRESPONDÊNCIA

MATHIAS ROBERTO LOCH

mathiasuel@hotmail.com
Departamento de Educação Física -

Centro de Educacao Fisica e Esporte

Universidade Estadual de Londrina

Rod. Celso Garcia Cid PR 445 -

Campus Universitario Londrina-PR,

Brasil. CEP: 86051-980 